\title{
RELAÇÕES MORFOMÉTRICAS PARA Araucaria angustifolia (Bertol.) Kuntze EM SANTA CATARINA
}

\author{
Danieli Regina Klein ${ }^{1 *}$, André Felipe Hess ${ }^{1}$, Sandra Mara Krefta ${ }^{1}$, Mushue Dayan Hampel Vieira Filho ${ }^{1}$, Lucas \\ Dalmolin Ciarnoscki ${ }^{2}$, Emanuel Arnoni Costa $^{3}$

\footnotetext{
${ }^{1}$ Universidade do Estado de Santa Catarina, Centro de Ciências Agroveterinárias, Departamento de Engenharia Florestal, Lages, Santa Catarina, Brasil - drkleinn@gmail.com*, andre.hess@udesc.br, sandra_krefta@gmail.com, mushuehampel@gmail.com.

${ }^{2}$ Universidade Federal do Paraná, Setor de Ciências Agrárias, Curitiba, Paraná, Brasil - ciarnoschifloresta @ gmail.com.
} \\ ${ }^{3}$ Universidade Federal de Santa Maria, Centro de Ciências Rurais, Santa Maria, Rio Grande do Sul, Brasil - emanuelarnonicost@gmail.com.
}

\begin{abstract}
Resumo
Conhecer a morfometria de uma espécie e descrever suas relações auxilia no planejamento e uso sustentável da floresta. O trabalho objetivou analisar relações morfométricas, a fim de ajustar modelos que representem 121 árvores de Araucaria angustifolia amostradas em dois sítios em São José do Cerrito, Planalto Catarinense. Foram mensurados: diâmetro à altura do peito (Dap), altura (h), altura de inserção de copa (hic), raio de copa (rc), comprimento de copa (cc), diâmetro de copa (dc), índice de abrangência (IA), índice de saliência (IS), grau de esbeltez (GE), formal de copa (FC), proporção de copa (Pc\%) e a posição sociológica (PS) de cada indivíduo. Foi usada correlação de Person para avaliar as relações entre variáveis morfométricas. Empregou-se a análise de covariância para verificar diferença de nível e inclinação nos ajustes dos modelos para os sítios. O ajuste dos modelos foi realizado pelos modelos lineares generalizados (MLG) nos campos de distribuição gama e normal, função de ligação identidade e logarítmica para correlações maiores ou iguais a 0,7. A análise de covariância demonstrou diferenças nas relações para cada sítio, indicando o ajuste de equações distintas. As equações demonstraram variações nas relações morfométricas pela mudança formadimensão de cada árvore. Os resultados mostram a modificação na forma da copa, a partir da posição social de cada árvore, além da influência diamétrica e do espaço ocupado por cada árvore na floresta, competição e condições do sítio. As relações morfométricas estabelecidas foram importantes para explicar a dinâmica de crescimento da espécie e a aplicação de intervenções silviculturais.
\end{abstract}

Palavras-chave: Morfometria; modelagem; manejo florestal.

\begin{abstract}
Morphometric relations for Araucaria angustifolia (Bertol.) Kuntze in Santa Catarina, Brazil. Knowing the morphometry of a species and describing its relations help us in the planning and in the sustainable use of the forest. The objective of this study was to analyze morphometric relationships in order to fit models representing 121 Araucaria angustifolia trees sampled at two sites in São José do Cerrito, Planalto Catarinense. The measures were: diameter at breast height (Dap), height (h), crown insertion height (hic), crown radius (rc), crown length (cc), crown diameter (dc), slenderness index (IS), degree of slenderness (GE), formal cup (HR), crown ratio (Pc\%) and sociological position (PS) of each individual. Person correlation was used to evaluate the relationships between morphometric variables. The covariance analysis was used to verify difference of level and slope in the adjustments of the models for the sites. The fit of the models was done by generalized linear models (MLG) in the gamma and normal distribution fields, identity and logarithmic link function for correlations greater than or equal to 0.7. The covariance analysis showed differences in the relations for each site, indicating the adjustment of different equations. The equations showed variations in morphometric relationships due to the shape-dimension change of each tree. The results show the modification in the shape of the canopy, from the social position of each tree, besides the diametrical influence and the space occupied by each tree in the forest, competition and site conditions. The established morphometric relationships were important to explain the growth dynamics of the species and the application of silvicultural interventions.
\end{abstract}

Keywords: Morphometry, modeling, forest management.

\section{INTRODUÇÃO}

Araucaria angustifolia (Bertol.) Kuntze é espécie característica da Floresta Ombrófila Mista. As condições atuais dos fragmentos de ocorrência natural da espécie no estado de Santa Catarina mostram que o desenvolvimento da espécie nesses remanescentes está comprometido pela falta de manejo. 
Um aspecto importante no manejo de espécies florestais é conhecer a morfometria das árvores, a qual é obtida por meio de informações coletadas a partir de variáveis como diâmetro à altura do peito (Dap), altura e dados relacionados à copa. As relações morfométricas, de acordo com Costa et al. (2016), possibilitam retratar as dimensões das árvores sem necessidade de identificar sua idade. Além disso, contribuem para propor intervenções silviculturais e planejamento florestal, sobretudo, quando se visa o uso sustentável das florestas.

Em um estudo sobre variáveis morfométricas e índices de competição, Hess et al. (2016) afirmaram que os remanescentes de floresta com A. angustifolia no sul do Brasil estão em competição e necessitam de intervenções silviculturais para estimular o crescimento das árvores remanescentes. Esses autores concluíram que a competição provoca mudanças na morfometria da copa e está correlacionada com o diâmetro à altura do peito, sítio, nível de competição entre as árvores e os recursos do ambiente. Os ajustes de relações morfométricas mostraram que isso provoca mudanças na dinâmica estrutural da floresta.

Minatti et al. (2016) indicaram que o intervalo de variação na forma das árvores é considerado pela variação em sua dimensão, bem como pelo nível de competição em que as árvores estavam sujeitas durante seu desenvolvimento. Com isso, o estudo das relações morfométricas e interdimensionais pode permitir a identificação da necessidade de intervenção na floresta.

Assim, a hipótese deste estudo se baseia na existência de variações na forma da copa e relações morfométricas de acordo com a dimensão, posição sociológica e sítio de ocorrência da espécie e, ainda, que essas variações podem ser explicadas por modelos de regressão. Para tanto, o objetivo do trabalho foi estabelecer correlações para as relações morfométricas e ajustar modelos de regressão com as variáveis fortemente correlacionadas para interpretar suas modificações de acordo com a posição sociológica e dimensão para $A$. angustifolia no Planalto Catarinense, Santa Catarina.

\section{MATERIAL E MÉTODOS}

Os dados foram coletados no município de São José do Cerrito em dois sítios amostrais de remanescentes da Floresta Ombrófila Mista com ocorrência natural de A. angustifolia no planalto serrano de Santa Catarina. Os solos da região são pouco profundos e caracterizados como Neossolos Litólicos e Cambissolos. O clima característico do município, conforme a classificação de Köppen, é do tipo Cfb, mesotérmico úmido, com chuvas bem distribuídas durante o ano e precipitação média anual de $1.400 \mathrm{~mm}$ na região. O município pertence à Bacia do Rio Canoas e do Rio Pelotas, com topografia suave-ondulada a forte ondulada (IBGE, 2012).

O primeiro sítio amostral localiza-se próximo a pastagens, com terreno pouco ondulado, e as árvores amostradas encontravam-se no interior da floresta. O segundo sítio trata-se de uma propriedade com terreno declivoso e com presença, em alguns pontos, de pedregosidade. Parte dos indivíduos avaliados estavam em área adjacente à floresta, enquanto que os demais foram coletados no interior da mesma.

Nesta pesquisa, foram amostradas 121 árvores de A. angustifolia, selecionadas aleatoriamente, seguindo o parâmetro de estudo de árvores individuais, sendo x no primeiro e y no segundo sítio. A suficiência amostral foi avaliada a partir da variável Dap para a determinação do número mínimo de árvores necessárias para abranger a variação do fragmento. Foi observado o valor suficiente de 22 árvores para o sítio 1, e 51 árvores para sítio 2, totalizando 73 árvores, ou seja, foi alcançado o critério quantitativo para suficiência amostral. As árvores foram classificadas em quatro classes diamétricas: classe 1, para indivíduos menores que $25 \mathrm{~cm}$ de Dap; classe 2, para Dap de 25 a 34 cm; classe 3, Dap de 35 a 44 cm; e classe 4, com árvores acima ou igual a $45 \mathrm{~cm}$ de Dap.

Há diferentes variáveis utilizadas para avaliar a morfometria de árvores. Neste trabalho, foi calculado o raio médio de copa em metros $(\overline{\mathrm{rc}}): \overline{\mathrm{rc}}=\sum_{\mathrm{i}=1}^{\mathrm{n}=4} r c * 4^{-1}$, representando a média de quatro projeções do raio de copa (rc), tomados nas posições Norte, Sul, Leste e Oeste; o diâmetro de copa em metros $(\mathrm{dc}): \mathrm{dc}=2 * \overline{\mathrm{rc}}$; o comprimento de copa em metros (cc), a diferença entre altura (h) e altura de início de copa (hic): cc =h-hic; a proporção de copa $(\mathrm{Pc} \%)$ : $\mathrm{Pc} \%=\left(c c * h^{-1}\right) * 100$; o índice de abrangência (IA): IA $=\left(\mathrm{dc} * \mathrm{~h}^{-1}\right)$; o índice de saliência (IS): IS $=\left(\mathrm{dc} * \operatorname{Dap}^{-1}\right)$; o grau de esbeltez $(\mathrm{GE}): \mathrm{GE}=\left(\mathrm{h} * \mathrm{Dap}^{-1}\right)$; e o formal de copa $(\mathrm{FC}): \mathrm{FC}=(\mathrm{dc} *$ $\left(c^{-1}\right)$.

Segundo Durlo e Denardi (1998), o cálculo dessas variáveis morfométricas explica em conjunto o espaço vital ocupado por cada árvore (indicado pelos índices IA, IS e dc) e fornece uma ideia do grau de concorrência em uma floresta, permitindo, ainda, inferir sobre sua estabilidade (resistência ao vento), indicada pelo grau de esbeltez, sua vitalidade e a produtividade de cada árvore (indicada pelas variáveis: comprimento de copa e proporção de copa).

Para avaliar a correlação existente entre as variáveis morfométricas, foi utilizada a correlação linear de Pearson $(r)$, considerando o nível de significância de $1 \%$, pois essas variáveis são fortemente correlacionadas, 
podendo ser expressas por relações lineares, como comprovadas por Durlo e Denardi (1998) e Costa et al. (2016).

As variáveis morfométricas com $r \geq 0,7$ foram submetidas à análise de covariância, visando identificar possíveis diferenças de nível (coeficiente $b_{0}$ ) e inclinação (coeficiente $b_{1}$ ) no ajuste de modelos para essas variáveis nos sítios de estudo. Foram consideradas as variáveis morfométricas e sua variabilidade como uma variável dependente que explica aspectos particulares do sítio, competição e dinâmica da forma das árvores. Foi adotado o modelo linear simples para a análise de covariância, a fim de evitar a diminuição dos graus de liberdade do resíduo de uma unidade e a perda do poder de ajuste do modelo (KAPS; LAMBERSON, 2004).

Os resíduos do ajuste dos modelos foram submetidos à análise das condicionantes de regressão (SCHNEIDER et al., 2009). Em caso de não atender essas condicionantes de regressão, os modelos foram ajustados empregando-se a técnica dos modelos lineares generalizados (MLGs) e testando-se os campos de distribuição aleatória Normal, Gama e as funções de ligação identidade e logarítmica. A acurácia do ajuste de modelos com MLG foi avaliada pelos critérios do Akaike (AIC), Bayesiano (BIC), Desvio (D), e análise gráfica dos resíduos, sendo que, quanto menor esses valores, melhor o ajuste do modelo (TURKMAN; SILVA, 2000). As análises estatísticas foram obtidas no Sistema de Análise Estatística (SAS, 2004).

\section{RESULTADOS}

A caracterização dos estratos de posição sociológica para os remanescentes de A. angustifolia indicou a seguinte estrutura diamétrica para as 121 árvores amostradas nos dois sítios estudados (Figura 1), em que foram determinadas quatro classes diamétricas que melhor representaram a distribuição dos indivíduos nos fragmentos. Houve maior número de indivíduos na classe 2 e menor na classe 1.

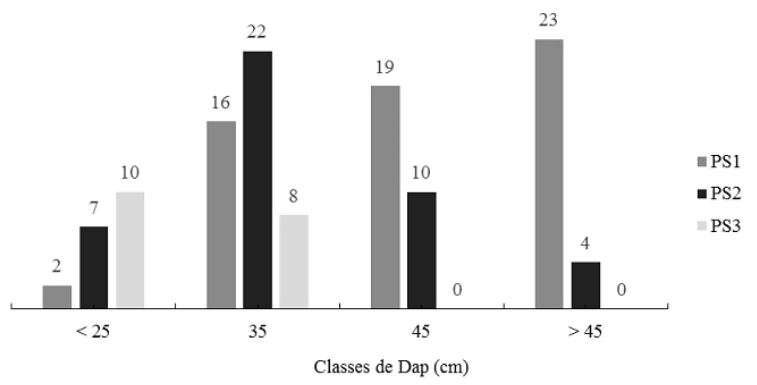

Figura 1. Distribuição diamétrica por posição sociológica de árvores de A. angustifolia em São José do Cerrito, SC, em que: PS1 (Dominante); PS2 (Codominante); PS3 (Dominada).

Figure 1. Diametric distribution by sociological position of A. angustifolia in São José do Cerrito, SC, Brazil, where: PS1 (Dominant); PS2 (Codominant); PS3 (Dominated).

As características morfométricas para as árvores observadas nos dois sítios de estudo estão apresentadas na Tabela 1. As araucárias amostradas apresentaram amplitude diamétrica de 15,92 a 97,08 cm entre as posições sociais. As árvores do sítio 1 foram, em média, mais altas que as araucárias do sítio 2.

Tabela 1. Amplitude das variáveis para A. angustifolia nos dois sítios amostrados no Planalto Catarinense. Table 1. Amplitude of the variables for A. angustifolia in two sites sampled in Planalto Catarinense, Brazil.

\begin{tabular}{cccccccccc}
\hline \multirow{2}{*}{ Variável } & \multirow{2}{*}{ PS } & \multicolumn{2}{c}{ Mínimo } & \multicolumn{2}{c}{ Médio } & \multicolumn{2}{c}{ Máximo } & \multicolumn{2}{c}{ CV\% } \\
\cline { 2 - 10 } & S1 & S2 & S1 & S2 & S1 & S2 & S1 & S2 \\
\hline \multirow{3}{*}{ Dap (cm) } & 1 & 25,78 & 23,24 & 38,92 & 53,69 & 54,43 & 97,08 & 19,96 & 38,01 \\
& 2 & 18,14 & 24,83 & 28,88 & 38,72 & 45,52 & 57,36 & 21,77 & 21,25 \\
& 3 & 15,92 & 19,74 & 22,28 & 25,8 & 31,83 & 32,15 & 29,32 & 15,49 \\
\hline \multirow{3}{*}{ h (m) } & 1 & 14 & 8,9 & 17,95 & 16,11 & 21,1 & 22,1 & 10,34 & 20,44 \\
& 2 & 14,7 & 10,3 & 17,29 & 15,74 & 21,9 & 19,9 & 11,46 & 16,23 \\
& 3 & 13,2 & 11 & 15,54 & 14,65 & 18,1 & 18,2 & 11,07 & 15,01 \\
\hline \multirow{2}{*}{ hic (m) } & 1 & 7,3 & 3,2 & 10,7 & 8,15 & 13,5 & 12,8 & 16,59 & 41,21 \\
& 2 & 8,2 & 4,6 & 11,28 & 9,15 & 14,4 & 13 & 13,84 & 30,68 \\
\hline cc (m) & 3 & 8,4 & 3,6 & 10,47 & 9,33 & 12,1 & 16,8 & 12,26 & 38,4 \\
\hline
\end{tabular}

FLORESTA, Curitiba, PR, v. 47, n. 4, p. 501 - 512, out. / dez. 2017.

Klein, D,R. et al.

ISSN eletrônico 1982-4688

DOI: $10.5380 /$ rf.v47i4.49667 


\begin{tabular}{cccccccccc} 
& 2 & 1,8 & 3 & 6,01 & 6,59 & 12,4 & 11,3 & 40,87 & 26,63 \\
& 3 & 3,5 & 0,9 & 5,07 & 5,33 & 6,8 & 8,3 & 24,08 & 42,67 \\
\hline \multirow{3}{*}{$\mathrm{Pc} \%$} & 1 & 20,59 & 23,53 & 39,96 & 50,42 & 59,38 & 79,11 & 26,57 & 32,24 \\
& 2 & 11,84 & 20,69 & 34,04 & 42,6 & 56,62 & 67,83 & 32,23 & 28,22 \\
& 3 & 26,22 & 5,08 & 32,48 & 37,78 & 41,21 & 67,27 & 18,63 & 47,21 \\
\hline \multirow{3}{*}{$\mathrm{dc}(\mathrm{m})$} & 1 & 5,45 & 4,8 & 7,63 & 11,13 & 10,85 & 20,5 & 17,77 & 38,04 \\
& 2 & 3,3 & 5,75 & 5,78 & 9,12 & 12,65 & 14,9 & 32,24 & 25,8 \\
& 3 & 3,55 & 5,2 & 5,53 & 6,82 & 10,35 & 10,1 & 41,24 & 22,17 \\
\hline \multirow{2}{*}{ IA } & 1 & 0,28 & 0,31 & 0,43 & 0,71 & 0,63 & 1,6 & 20,54 & 42,05 \\
& 2 & 0,21 & 0,29 & 0,34 & 0,59 & 0,83 & 0,79 & 36,24 & 24,51 \\
& 3 & 0,23 & 0,29 & 0,35 & 0,47 & 0,57 & 0,66 & 32,47 & 24,34 \\
\hline \multirow{3}{*}{ IS } & 1 & 16,77 & 14,2 & 19,82 & 20,97 & 26,23 & 27,93 & 12,34 & 16,73 \\
& 2 & 15,55 & 17,71 & 20,07 & 23,67 & 34,86 & 30,52 & 22,85 & 16,88 \\
& 3 & 14,48 & 20,17 & 25,28 & 26,49 & 34,96 & 34,87 & 29,74 & 15,41 \\
\hline \multirow{2}{*}{ GE } & 1 & 30,46 & 13,55 & 47,68 & 33,55 & 73,69 & 63,55 & 20,1 & 35,11 \\
& 2 & 37,65 & 30,67 & 62,06 & 42,06 & 82,67 & 69,28 & 19,78 & 25,07 \\
& 3 & 51,84 & 45,1 & 73,36 & 57,52 & 93,68 & 73,98 & 20,92 & 16,44 \\
\hline \multirow{2}{*}{ FC } & 1 & 0,64 & 0,6 & 1,16 & 1,64 & 2,51 & 4,8 & 38,31 & 65,49 \\
& 2 & 0,52 & 0,83 & 1,24 & 1,5 & 7,03 & 3,75 & 102,79 & 46,1 \\
& 3 & 0,79 & 0,64 & 1,08 & 1,88 & 1,57 & 7,67 & 26,11 & 5,2 \\
\hline
\end{tabular}

Legenda: PS: posição sociológica; CV\%: coeficiente de variação; S1: sítio 1; S2: sítio 2; Dap: diâmetro à altura do peito (cm); h: altura total $(\mathrm{m})$; hic: altura de inserção de copa $(\mathrm{m})$; dc: diâmetro de copa $(\mathrm{m})$; cc: comprimento de copa (m); Pc\%: proporção de copa (\%); GE: grau de esbeltez; FC: formal de copa; IA: índice de abrangência e IS: índice de Saliência.

A correlação de Pearson, demonstrada na Tabela 2, permitiu inferir sobre o grau de associação entre as variáveis morfométricas, em que se obteve 15 correlações significativas. Porém, para o estudo, foi utilizado como critério de modelagem o uso das cinco maiores correlações, com a finalidade de verificar como essas variáveis refletem no desenvolvimento das árvores de A. angustifolia.

Tabela 2. Correlação de Pearson e probabilidades para as variáveis de A. angustifolia no Planalto Catarinense.

Table 2. Pearson correlation and probabilities for the variables of A. angustifolia in Planalto Catarinense, Brazil.

\begin{tabular}{llllllllll}
\hline & Dap & h & hic & cc & Pc\% & dc & IA & IS & GE \\
\hline h & $0,18^{\mathrm{ns}}$ & - & - & - & - & - & - & - & - \\
hic & $-0,02^{\mathrm{ns}}$ & $0,60^{*}$ & - & - & - & - & - & - & - \\
$\mathbf{c c}$ & $0,21^{\mathrm{ns}}$ & $0,40^{*}$ & $-0,50^{*}$ & - & - & - & - & - & - \\
Pc\% & $0,12^{\mathrm{ns}}$ & $-0,12^{\mathrm{ns}}$ & $-0,86^{*}$ & $0,85^{*}$ & - & - & - & - & - \\
$\mathbf{d c}$ & $0,87^{*}$ & $0,04^{\mathrm{ns}}$ & $-0,07^{\mathrm{ns}}$ & $0,12^{\mathrm{ns}}$ & $0,08^{\mathrm{ns}}$ & - & - & - & - \\
$\mathbf{I A}$ & $0,75^{*}$ & $-0,34^{\mathrm{ns}}$ & $-0,29^{\mathrm{ns}}$ & $-0,04^{\mathrm{ns}}$ & $0,15^{\mathrm{ns}}$ & $0,91^{*}$ & - & - & - \\
$\mathbf{I S}$ & $-0,23^{\mathrm{ns}}$ & $-0,32^{\mathrm{ns}}$ & $-0,13^{\mathrm{ns}}$ & $-0,19^{\mathrm{ns}}$ & $-0,06^{\mathrm{ns}}$ & $0,24^{\mathrm{ns}}$ & $0,31^{\mathrm{ns}}$ & - & - \\
GE & $-0,82^{*}$ & $0,17^{\mathrm{ns}}$ & $0,32^{\mathrm{ns}}$ & $-0,19^{\mathrm{ns}}$ & $-0,28^{\mathrm{ns}}$ & $-0,77^{*}$ & $-0,77^{*}$ & $0,14^{\mathrm{ns}}$ & - \\
FC & $0,37^{\mathrm{ns}}$ & $-0,17^{\mathrm{ns}}$ & $0,31^{\mathrm{ns}}$ & $-0,55^{*}$ & $-0,51^{*}$ & $0,52^{*}$ & $0,57^{*}$ & $0,30^{\mathrm{ns}}$ & $-0,35^{\mathrm{ns}}$ \\
\hline
\end{tabular}

Legenda: Dap: diâmetro à altura do peito $(\mathrm{cm})$; h: altura total $(\mathrm{m})$; hic: altura de inserção de copa $(\mathrm{m})$; Cc: comprimento de copa (m); Pc\%: proporção de copa (\%); dc: diâmetro de copa (m); IA: índice de abrangência; IS: índice de saliência; GE: grau de esbeltez; FC: formal de copa; *: significativo a $1 \%$ de significância estatística; ${ }^{\text {ns }}$ : não significativo a $1 \%$ de significância estatística.

A análise de covariância demonstrou a existência de diferenças entre os níveis e inclinação das linhas de regressão para as relações morfométricas (Tabela 3). Assim, com base na análise de covariância, houve necessidade de regressões distintas para as relações interdimensionais. 
Tabela 3. Análise de covariância para diâmetro de copa (dc), proporção de copa (Pc\%), grau de esbeltez (GE) e índice de abrangência (IA) em função de variáveis correlacionadas para A. angustifolia no Planalto Catarinense.

Table 3. Covariance analysis for crown diameter (dc), crown percentage (Pc\%), degree of slenderness (GE) and index of comprehensiveness (IA) as function of correlated variables of A. angustifolia in Planalto Catarinense, Brazil.

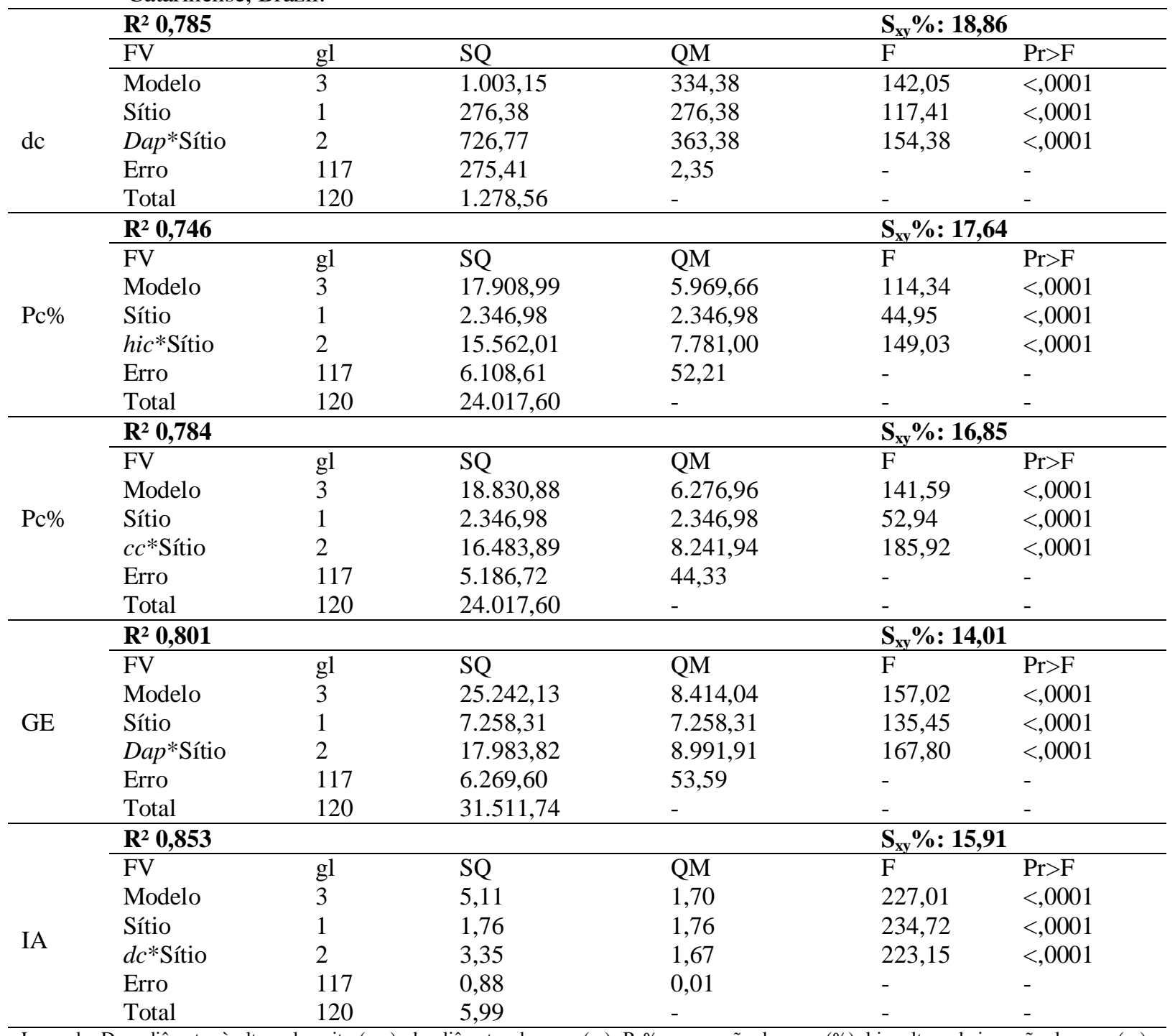

Legenda: Dap: diâmetro à altura do peito $(\mathrm{cm})$; dc: diâmetro de copa $(\mathrm{m})$; Pc\%: proporção de copa $(\%)$; hic: altura de inserção de copa (m); cc: comprimento de copa $(\mathrm{m})$; GE: grau de esbeltez; IA: índice de abrangência; $\mathrm{R}^{2}$ : coeficiente de determinação; $\mathrm{S}_{\mathrm{yx}}$ : erro padrão da estimativa em porcentagem; FV: fonte de variação; gl: graus de liberdade; SQ: soma de quadrados; QM: quadrado médio; F: valor da estatística F; Prob.>F: probabilidade de F.

Após testar os MLGs nos três campos aleatórios, Normal, Poisson e Gama, e nas funções de ligação identidade e logarítmica, obteve-se o melhor modelo com base nos critérios de AIC, BIC e desvio para cada relação avaliada, conforme Tabela 4.

Tabela 4. Valores dos ajustes de MLG para as relações morfométricas estabelecidas para A. angustifolia no Planalto Catarinense.

Table 4. MLG adjustments values for morphometric relationships established for A. angustifolia of Planalto Catarinense, Brazil.

\begin{tabular}{|c|c|c|c|c|c|c|c|}
\hline Dolocก̃ & Sítio & Parâmetros & Distribuis̃̃ & Função de & & & \\
\hline Relaçao & SIt10 & $\Phi_{0} \quad \Phi_{1}$ & DistrıDüçao & ligação & AlC & BIC & Desvio \\
\hline
\end{tabular}

FLOREST A, Curitiba, PR, v. 47, n. 4, p. 501 - 512, out. / dez. 2017.

Klein, D,R. et al.

ISSN eletrônico 1982-4688

DOI: $10.5380 /$ rf.v47i4.49667 


\begin{tabular}{ccccccccc}
\multirow{2}{*}{$\mathrm{dc}=\mathrm{f}($ Dap $)$} & 1 & 1,079 & 0,024 & Gama & Log & 200,86 & 204,95 & 2,03 \\
& 2 & 1,809 & 0,180 & Gama & Id & 218,74 & 222,64 & 1,66 \\
\hline \multirow{2}{*}{$\mathrm{Pc} \%=\mathrm{f}(\mathrm{hic})$} & 1 & 91,063 & $-4,976$ & Gama & Id & 431,76 & 435,84 & 0,04 \\
& 2 & 87,743 & $-4,788$ & Gama & Id & 418,78 & 422,87 & 0,04 \\
\hline \multirow{2}{*}{$\mathrm{Pc} \%=\mathrm{f}(\mathrm{cc})$} & 1 & 5,942 & 4,763 & Gama & Id & 328,02 & 332,10 & 0,01 \\
& 2 & 0,272 & 6,541 & Gama & Id & 410,51 & 414,60 & 0,04 \\
\hline \multirow{2}{*}{$\mathrm{GE}=\mathrm{f}(\mathrm{Dap})$} & 1 & 1,027 & $-0,014$ & Normal & Id & $-166,22$ & $-162,13$ & 0,25 \\
& 2 & $-0,106$ & $-0,019$ & Normal & Log & $-133,36$ & $-129,46$ & 0,32 \\
\hline \multirow{2}{*}{$\mathrm{IA}=\mathrm{f}(\mathrm{dc})$} & 1 & $-1,844$ & 0,129 & Normal & $\log$ & $-166,22$ & $-162,13$ & 0,12 \\
& 2 & $-1,312$ & 0,082 & Normal & $\log$ & $-133,36$ & $-129,46$ & 0,64 \\
\hline
\end{tabular}

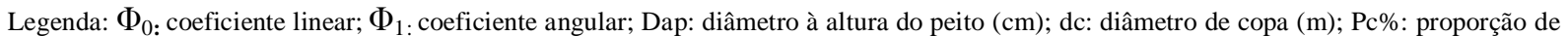
copa (\%); hic: altura de inserção de copa (m); Cc: comprimento de copa (m); GE: grau de esbeltez; IA: índice de abrangência; BIC: critério bayesiano; AIC: critério de Akaike; Desvio: função desvio do modelo; Log: Logarítmica; Id.: Identidade.

Na Figura 2, está apresentada a dispersão de resíduos dos modelos selecionados pelo MLG para cada relação analisada. Pode-se observar distribuição residual adequada entre as variáveis amostradas.
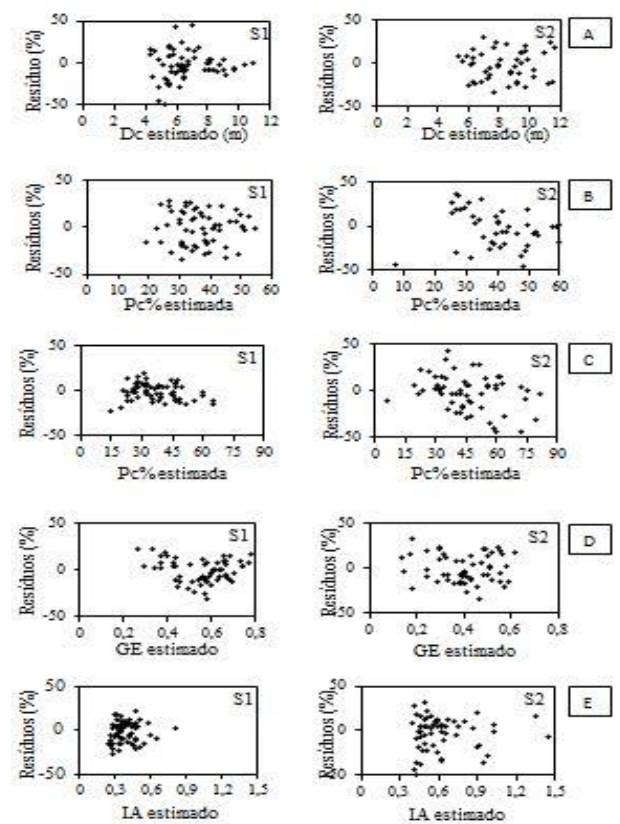

Figura 2. Dispersão dos resíduos para o MLG das relações de A. angustifolia no Planalto Catarinense. Em que: A: relação dc com Dap; B: relação Pc\% com hic; C: relação Pc\% com cc; D: relação GE com Dap; E: relação IA com dc; S1: sítio 1; S2: sítio 2; dc: diâmetro de copa; Pc\%: Proporção de copa; GE: grau de esbeltez; IA: índice de abrangência.

Figure 2. Distribution of the residues to the MLG of the ratios of A. angustifolia in Planalto Catarinense, Brazil. In which: A: dc relation with Dap; B: relation Pc\% with hic; C: relation Pc\% with cc; D: GE relation with Dap; E: relation IA with Dc; S1: site 1; S2: site 2; Dc: crown diameter; Pc\%: Percentage cup; GE: degree of slenderness; IA: index of comprehensiveness.

Para avaliar o comportamento dos dados, traçou-se a linha estimativa da variável dependente, juntamente com os valores observados para cada relação avaliada, os quais estão apresentados na Figura 3. 

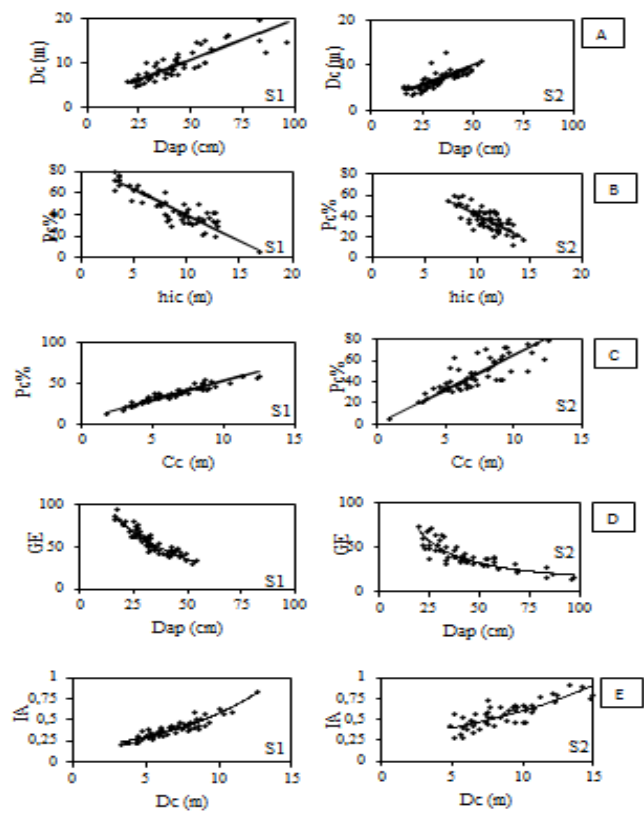

Figura 3. Dispersão dos valores observados e estimados das relações de A. angustifolia no Planalto Catarinense, Brasil. Em que: A: relação dc com Dap; B: relação Pc\% com hic; C: relação Pc\% com cc; D: relação GE com Dap; E: relação IA com dc; S1: sítio 1; S2: sítio 2; Dap: diâmetro à altura do peito; hic: altura de inserção de copa; cc: comprimento de copa; dc: diâmetro de copa; Pc\%: proporção de copa; GE: grau de esbeltez; IA: índice de abrangência.

Figure 3. Dispersion of the values observed on the line adjusted by the A. angustifolia in Planalto Catarinense, Brazil. In which: A: dc relation with Dap; B: relation Pc\% with hic; C: relation Pc\% with cc; D: GE relation with Dap; E: relation IA with dc; S1: site 1; S2: site 2; dc: crown diameter; Pc\%: Percentage cup; GE: degree of slenderness; IA: index of comprehensiveness.

\section{DISCUSSÃO}

Os resultados mostraram uma distribuição regular, mesmo com maior número de indivíduos nas maiores classes de diâmetro, sendo a classe 1, abaixo de $25 \mathrm{~cm}$, a que exibiu menor quantidade de indivíduos. Lamprecht (1962) afirmou que uma distribuição diamétrica regular favorece a existência e sobrevivência das espécies regenerantes.

Estudos realizados por Chassot et al. (2011) e Costa (2011) com A. angustifolia constataram que árvores com copas vigorosas e diâmetros maiores ocupam o estrato superior dentre as demais árvores, sendo que a maior incidência de luz favorece seu desenvolvimento. Assim, a morfometria da copa se modifica conforme o espaço ocupado pela árvore, beneficiando árvores do estrato dominante pelos recursos que competem.

As médias exibidas na Tabela 1 para o Dap do sítio 1, nas diferentes posições sociológicas, foram menores que as médias do sítio 2 e o contrário ocorreu para variável $h$. Conforme as condições de desenvolvimento e competição por recursos, os maiores valores de Dap e $\mathrm{h}$ foram de árvores classificadas como dominantes (PS1), que é uma tendência natural da espécie, a qual possui a característica de se sobressair no dossel da floresta.

A hic foi maior em PS2 para o sítio 1 e PS3 para o sítio 2. Para Costa et al. (2016), as árvores dominantes com baixo hic indicam redução do crescimento em $\mathrm{h}$ e revelam que a mortalidade dos ramos na base da copa é maior que o aumento do cc e hic, o que corrobora com os dados apresentados para o sítio 1, indicando, assim, competição.

Para Cc, os valores foram maiores na PS1 e menores na PS3 para ambos os sítios. Essa situação de cc menores indica a ocorrência de auto desrama nesses indivíduos. O dc para a A. angustifolia do sítio 2 foi maior do que do sítio 1, sendo que as árvores de PS1 apresentaram dimensões de copas superiores para os dois sítios. Maiores valores de dc sugerem maior espaço lateral para crescimento e desenvolvimento das árvores, o que favorece para que estas alcancem o dossel da floresta, demonstrando capacidade de competição por luz e recursos. 
A variação média da Pc\% sugere que entre 32 e $51 \%$ da altura total das árvores é ocupada pela copa. Essa variável é um indicador de vitalidade e, quanto maior seu valor, maior a produtividade da árvore. Nesse estudo, a maior Pc\% foi de 79,11\% e a menor, 5,08\%. Para Roman et al. (2009), essa amplitude advém da diferença da intensidade de competição a que cada árvore é submetida, estando associada às dimensões dos indivíduos.

O IA revelou-se maior para árvores PS1 e menor para PS3, sendo maior no sítio 2. Como esse índice se constitui da razão entre o dc e a h, valores mais altos indicam que a h das árvores do sítio 2 são, em média, menores que as do sítio 1, com dc maiores para o sítio 2. Roveda et al. (2012) afirmaram que, para um povoamento onde o manejo é baseado na h das árvores, o IA é critério para tomada de decisão em um plano de desbaste. Assim, conforme o aumento da h, há necessidade de um espaço maior entre as árvores para desenvolvimento em Dap.

O IS retratou uma situação semelhante para ambos os sítios em estudo e para os níveis sociológicos. Esse índice representa que, em média, as árvores têm diâmetro de copa, aproximadamente, de 19,82 a 26,49 vezes maior quando comparado ao Dap. O IS pode ser usado como parâmetro indicador do espaço necessário para cada árvore ao ser atingido determinado diâmetro, ou pode indicar o número de árvores adequado de acordo com um diâmetro de interesse estabelecido.

Mattos (2007) ressaltou que, quando se prediz o manejo de um povoamento pelo diâmetro alcançado por seus componentes e não pela idade, o número máximo de árvores por unidade de área pode ser calculado pelo IS à medida que as árvores crescem, caso haja uma correlação significativa entre esse índice e o Dap.

O GE, em cada posição sociológica, foi maior para o sítio 1 em comparação com o sítio 2, com variação média de 73,36 a 33,55. Esse índice caracteriza a estabilidade das árvores, ou seja, quanto mais alto o grau de esbeltez, mais instável é a árvore, uma vez que essa será alta, porém, fina. Assim, maiores valores para GE apontam que as árvores estão crescendo mais em altura do que em diâmetro.

Estudos realizados com A. angustifolia por Loiola (2016) e Minatti (2015) resultaram em valores médios para GE de 40,6 e 50,0, respectivamente, o que aponta maior competição por espaço-recurso e árvores ocupando estratos de codominante e dominada, resultando em baixa vitalidade e eficiência em espaço de crescimento.

O FC revelou valores maiores para árvores PS2 no sítio 1 e para árvores PS3 no sítio 2, com amplitude de 0,52 a 7,67. Esse gradiente, conforme Roveda et al. (2012), indica que, na população, há árvores com baixo FC, ou seja, copas esbeltas e, também, com copas mais achatadas e com dc superior a cinco vezes o seu comprimento, uma vez que FC faz inferência sobre a relação entre diâmetro de copa e comprimento de copa. Portanto, entre árvores com mesmo diâmetro, alcançará maior produtividade aquela que apresentar menor valor de FC. Assim, é possível utilizar esse índice na seleção de árvores para desbaste, onde serão retiradas aquelas de maior FC.

A análise de correlação de Pearson (Tabela 2) mostrou que à medida que o Dap aumentou, os valores de dc e FC também aumentaram, uma vez que essas correlações foram positivas. No entanto, valores para IS e GE diminuíram, pois tiveram correlação negativa com o Dap. O Dap com dc apresentou valor de correlação de 0,87 e o Dap com GE de -0,82. A hic apresentou correlação positiva com a h $(0,60)$, indicando árvores com menor manto de copa, maior dimensão, idade e posição sociológica de dominante e, consequentemente, menor Pc\% e menores taxas de crescimento, como demonstra a relação negativa entre Pc\% com hic $(-0,86)$.

Para as relações interdimensionais, foram selecionadas as cinco variáveis que tinham maiores correlações, sendo elas a relação entre Dap com dc $(0,87)$, Pc\% com Cc $(0,85)$ e dc com IA $(0,91)$, com correlações positivas. Para as correlações negativas, foram selecionadas as relações Dap com GE $(-0,82)$ e Pc\% com hic $(-0,86)$.

O Pc\% apresentou correlação negativa com hic $(-0,86)$ e positiva com o cc $(0,85)$. Curto $(2015)$, em um estudo realizado com A. angustifolia na Floresta Nacional (FLONA) de Açungui (PR), encontrou uma correlação de 0,47 para relação Pc\% e hic, e 0,90 para Pc\% e Cc. Valores discrepantes para relação entre Pc\% e hic referem-se a fatores genéticos, qualidade de sítio, grau de competição e condições biológicas e edafoclimáticas.

As relações avaliadas pela análise de covariância, apresentadas na Tabela 3, comprovaram existir diferença entre os níveis e inclinações das linhas de regressão para dc, Pc\%, GE e IA para sítio e interação*sítio com probabilidade menor que 0,0001 . Houve necessidade de regressões distintas para as relações avaliadas, sendo que as equações obtidas alcançaram $\mathrm{R}^{2}$ de 0,746 a 0,853 e erro padrão da estimativa de 14,01 a $18,86 \%$.

$\mathrm{Na}$ análise de covariância, foram ajustados os modelos para cada sítio separadamente, com uso dos modelos lineares generalizados (MLG), que são uma extensão dos modelos lineares, em que os dados não precisam contemplar os condicionantes de regressão, como ocorrido no presente trabalho. Além disso, trabalhos com o uso de MLG vêm sendo empregados na área florestal, como o de Hess et al. (2015). Com base nos critérios apresentados na Tabela 4 para relação do de com Dap no sítio 1, o melhor modelo foi Gama-logarítmico 
e, para o sítio 2, foi Gama-identidade, os quais apresentaram menor valor de Desvio, BIC e AIC. Para a relação da Pc\% com hic, o modelo Gama-identidade foi o melhor modelo para os dois sítios.

Na relação do Pc\% com o cc, o melhor modelo foi Gama-identidade para os dois sítios. Para avaliar a relação entre o GE e o Dap, o melhor modelo para o sítio 1 foi Normal-identidade e para o sítio 2 foi Normallogarítmica. Para relação de IA com dc, para os dois sítios, o melhor modelo foi Normal-logarítmica.

A análise gráfica dos resíduos também foi utilizada para a seleção dos modelos. Observando-se a dispersão dos resíduos para as relações avaliadas, notou-se que não houve tendência nos resíduos, conforme se observa na Figura 2. A variação observada em alguns valores demonstra a relação com as características ontogenéticas da espécie, ou seja, o desenvolvimento que ocorre nas árvores é dependente do sítio e competição. O comportamento das linhas dos valores estimados sobre os valores observados, representados por pontos (Figura 3), apresentou-se sem tendência discrepante. Verificou-se forte relação entre as variáveis analisadas, sendo positiva para a relação entre dc com Dap, Pc\% com cc e IA com dc, e negativa para Pc\% com hic e GE com Dap.

A relação de dc com Dap indicou que, à medida que a árvore aumentou em Dap, aumentou o diâmetro de copa; ao mesmo passo que, quanto maior foi o cc, maior foi a Pc\%, característica de árvores de menor diâmetro, mas com maior potencial fotossintético. Assim, a dinâmica de desenvolvimento da forma da árvore apontou que, à medida que aumentou a hic, diminuiu a Pc\% e aumentou sua dimensão, demonstrando árvores de estratos superiores e copas em forma de umbela, ou ainda, que estão em intensa competição. Da mesma forma, à medida que aumentou o Dap, o GE diminuiu, exibindo maior altura total, maior captação de luz e melhor desenvolvimento em diâmetro. Todas essas relações são indicadoras do momento de intervenção silvicultural na floresta.

Além disso, os indivíduos amostrados apresentaram características de desenvolvimento diferente entre as áreas. Assim, demonstraram que a possibilidade de intervir para reduzir competição e favorecer o crescimento dos fragmentos garante a estabilidade da floresta. Do mesmo modo, os modelos gerados auxiliarão nas intervenções, servindo como prognósticos de possíveis situações.

Nesse contexto, autores como Nascimento et al. (2010), Sanquetta et al. (2013) e Costa et al. (2014) também observaram forte relação entre o Dap com o dc e GE, pois, à medida que as árvores crescem em Dap, a dimensão de suas copas também aumenta, diminuindo, assim, o GE da árvore; ou seja, seu desenvolvimento é favorecido, garantindo sua posição no estrato dominante da floresta.

\section{CONCLUSÕES}

Conforme os resultados, pode-se concluir que:

- As relações morfométricas avaliadas demonstraram que as características de copa da espécie foram influenciadas pelo diâmetro e pelo espaço ocupado por cada árvore no ambiente da floresta.

- O diâmetro à altura do peito foi uma variável fortemente relacionada com a dimensão da copa das árvores, assim como sua altura total.

- No ajuste dos modelos, pôde-se observar a forte influência da competição nas árvores amostradas, sendo que a variação entre elas se referiu às suas características de desenvolvimento na floresta, como o espaço e condiçõos do sítio.

\section{REFERÊNCIAS}

CHASSOT, T.; FLEIG, F. D.; FINGER, C. A. G.; LONGHI, S. J. Modelos de crescimento em diâmetro de árvores individuais de Araucaria angustifolia (Bertol.) Kuntze em floresta ombrófila mista. Ciência Florestal, Santa Maria, v. 21, n. 2, p. 303-313, 2011. DOI: http://dx.doi.org/10.5902/198050983234 .

COSTA, E. A. Influência de variáveis dendrométricas e morfométricas no incremento diamétrico de $\boldsymbol{A}$. angustifolia (Bertol.) Kuntze, Lages, SC. Dissertação (Mestrado). 148f. Universidade Federal de Santa Maria, Santa Maria - RS. 2011.

COSTA, E. A.; FINGER, C. A. G.; MARANGON G. P.; CUBAS, R.; LONGHI, R. V. Relação entre o diâmetro de copa e o diâmetro à altura do peito de Araucaria angustifolia (Bertol.) Kuntze, Lages, SC. In... Simpósio Brasileiro de Pós-graduação em Ciências Florestais. Recife-PE, p. 696-700, 2014. 
COSTA, E. A.; FINGER, C. A. G.; FLEIG, F. D. Influência da posição social nas relações morfométricas de Araucaria angustifolia. Ciência Florestal, Santa Maria, v. 26, n. 1, p. 225-234, 2016. DOI: http://dx.doi.org/10.5902/1980509821116.

DURLO, M. A.; DENARDI, L. Morfometria de Cabralea canjerana, em mata secundária nativa do Rio Grande do Sul. Ciência Florestal, v. 8, n. 1, p. 55-66, 1998. DOI: http://dx.doi.org/1580232.

IBGE - Instituto Brasileiro de Geografia e Estatística. Manual técnico da vegetação brasileira. Rio de Janeiro, 2012. $271 \mathrm{p}$.

HESS, A. F.; CIANORSCHI, L.; SILVESTRE, R.; SCARIOT, R.; RICKEN, P. Aplicação dos modelos lineares generalizados para estimativa do crescimento em altura. Pesquisa Florestal Brasileira. v. 35, n.84, p. 427-433. 2015. DOI: http://dx.doi.org/10.4336/2015.pfb.35.84.604

HESS, A. F.; LOIOLA, T. M.; SOUZA, I. A.; NASCIMENTO, B. Morphometry of the crown of Araucaria angustifolia in natural sites in southern Brazil. Bosque. 37(3): 603-611, 2016. DOI: 10.4067/S071792002016000300017.

KAPS, M., LAMBERSON, W. R. Biostatistics for Animal Science. CABI Publishing, London, UK, 2004.

LAMPRECHT, H. Ensayo sobre unos métodos para el Análisis Estructural de los bosques tropicales. Acta Científica Venezolana, 13 (2): 57-65, 1962.

LOIOLA, T. M.; Manejo da paisagem em fragmentos de floresta de A. angustifolia no sul do Brasil com base no incremento diamétrico. Dissertação (Mestrado). 136f. Universidade do Estado de Santa Catarina. Lages - SC. 2016.

MATTOS, R. B. Produtividade e incremento de Cabralea canjerana (Vell.) Mart., Cedrela fissilis Vell. e Cordia trichotoma (Vell.) Arrab. Ex Steud., em floresta nativa no Rio Grande do Sul. Tese (Doutorado). 105f. Universidade Federal de Santa Maria, Santa Maria - RS. 2007.

MINATTI, M. Dendrocronologia aplicada na geração de modelos de crescimento biométricos e ambientais para Araucaria angustifolia na fitorregião do Planalto Serrano, SC. Dissertação (Mestrado). 129f. Universidade do Estado de Santa Catarina. Lages - SC. 2015.

MINATTI, M.; HESS, A. F.; RICKEN, P.; LOIOLA, T. M.; SOUZA, I. A. Shape and size relationships of Araucaria angustifolia in South Brazil. African Journal of Agricultural. Vol. 11(41), p. 4121-4127, 2016. DOI: 10.5897/AJAR2016.11220.

NASCIMENTO, R. G. M.; MACHADO, S. do M.; FIGUEIREDO, D. J. AUGUSTYNCZIK, A.L.D.; CAVALHEIRO, .R Relações dendrométricas de Araucaria angustifolia. Pesquisa Florestal Brasileira (Nota científica). Colombo, v. 30, n. 64, p.369-374, 2010. DOI: 10.4336/2010.pfb.30.64.369.

ROMAN, M.; BRESSAN, D. A.; DURLO, M. A. Variáveis morfométricas e relações interdimensionais para Cordia trichotoma (Vell.) Arráb. ex Steud. Ciência Florestal, Santa Maria, v. 19, n. 4, p. 473-480, 2009. DOI: http://dx.doi.org/10.5902/19805098901.

ROVEDA, M.; DALGAllo, B.; DIAS, A. N.; FIGUEIREDO FILHO, A.; MULLER, C. S.. Morfometria de Araucaria angustifolia (Bertol.) Kuntze na Floresta Nacional de São Francisco de Paula - RS. In: IV Congresso Florestal Paranaense, 2012, Curitiba. IV Congresso Florestal Paranaense, 2012.

SANQUETTA, C. R.; DALlA CORTE, A. P.; ROGLIN, A.; PIMENTEL, A. Relações diâmetro-altura para espécies lenhosas em um fragmento de Floresta Ombrófila Mista no Sul do Paraná. Iheringia, Sér. Bot., Porto Alegre, v. 68, n. 1, p. 103-114, 2013.

SAS Institute. The SAS System for Windows. Cary: SAS Institute. 2004. 
SCHNEIDER, P. R.; SCHNEIDER, P. S. P.; SOUZA, C. A. M. Análise de regressão aplicada à engenharia florestal. Santa Maria: FACOS-UFSM, 2009. 294 p

TURKMAN, M. A. A.; SILVA, G. L. Modelos Lineares Generalizados - da teoria à prática. Universidade de Lisboa, 2000. Disponível em: http://docentes.deio.fc.ul.pt/maturkman/mlg.pdf. Acesso em maio de 2016. 
FLORESTA, Curitiba, PR, v. 47, n. 4, p. 501- 512, out/dez. 2017. Klein,D,R. et al. 